\title{
Patient-Specific Operative Planning for Aorto-Femoral Reconstruction Procedures
}

\author{
Nathan Wilson ${ }^{1}$, Frank R. Arko ${ }^{2}$, and Charles Taylor ${ }^{3}$ \\ ${ }^{1}$ Stanford University, Clark Center E372, Stanford, CA, 94305-5431 \\ nwilsonestanford. edu \\ ${ }^{2}$ Division of Vascular Surgery, Suite H-3600, Stanford University Medical Center, \\ Stanford, California 94305-5642 \\ farko@stanford.edu \\ ${ }^{3}$ Stanford University, Clark Center E350B, Stanford, CA 94305-5431 \\ taylorcalstanford.edu
}

\begin{abstract}
Traditionally, a surgeon will select a procedure for a particular patient based on past experience for patients with a similar state of disease. The experience gained from this patient will be selectively used when treating the next patient with similar symptoms. In this work, a surgical planning system was developed enabling a vascular surgeon to create and test alternative operative plans prior to surgery for a given patient. One dimensional and three dimensional hemodynamic (i.e. blood flow) simulations were performed for rest and exercise for operative plans for two aorto-femoral bypass patients and compared with actual postoperative data. The information that can be obtained from one-dimensional (volume flow distribution and pressure losses) and threedimensional (wall shear stress) hemodynamic simulation may be clinically relevant to vascular surgeons planning interventions.
\end{abstract}

\section{Introduction}

Atherosclerosis is a degenerative vascular disease of clinical significance which leads to a narrowing of the flow lumen preventing adequate flow to tissues and organs. In symptomatic patients with occlusive disease of the lower extremities, the infra-renal abdominal aorta and the iliac arteries are the most common sites of obliterative atherosclerosis [1]. Many patients asymptomatic under resting conditions experience ischemic pain under exercise conditions. The primary objective of a surgical intervention for aortoiliac occlusive disease is to restore adequate flow to the lower extremities under a range of physiologic states including rest and exercise. While volumetric flow distribution is of paramount concern initially following a surgical intervention, other quantities such as high particle residence time and low mean wall shear stress are theorized to be flow-related factors in disease progression and impact the long term efficacy of a surgical intervention.

Several major classes of treatment exist for aortoiliac occlusive disease. One technique that may be appropriate for localized occlusive disease is to use catheterbased endoluminal therapies such as angioplasty and stenting. The use of angioplasty 
and stenting has received considerable attention recently due to probable cost savings and decreased morbidity compared to traditional open surgical interventions. The most commonly used procedure for severe cases is direct anatomic surgical reconstruction and is the focus of this work presented here. Direct surgical reconstruction refers to the insertion of grafts replacing or providing alternative pathways to flow through the infra-renal aorta and iliac arteries.

Currently, surgery planning for the treatment of vascular disease involves acquiring diagnostic imaging data to assess the extent of aortoiliac disease in a given patient. The surgeon then relies on his/her past experience, personal bias, and previous surgical training to create a treatment strategy. Initially proposed by Taylor [2], a new paradigm of Simulation-Based Medical Planning (SBMP) for vascular disease has been proposed to utilize computational methods to evaluate alternative surgical options prior to treatment using patient-specific models of the vascular system. Blood flow (hemodynamic) simulations enable a surgeon to see the flow features resulting from a proposed operation and to determine if they pose potential adverse effects such as increased risk of atherosclerosis and thrombus formation.

\section{Methods}

There are several major steps in the SBMP process for vascular surgical applications [3]. First, patient-specific preoperative geometric models from medical imaging data are created by a technician. Additional imaging processing may occur to obtain physiologic information. This process can take several hours. Next, geometric models representing several surgical alternatives to be evaluated are constructed under the guidance of the attending vascular surgeon. This usually takes on the order of an hour. One and three-dimensional numerical simulations are then performed on the different surgical interventions. Finally, clinically relevant analysis results are then visualized and interpreted. The remaining part of this section summarizes some the important considerations.

\subsection{Patient-Specific Geometric Model Construction and Surgical Planning}

Magnetic Resonance Imaging (MRI) is a particularly useful technique for SBMP because it can provide both physiologic and volumetric geometric information. In the case studies presented here, the medical imaging data was obtained using a GE Signa 1.5T MRI scanner (General Electric, Milwaukee, WI, USA). Ideally, the scanner creates linearly varying gradients of the magnetic field across the image volume. However, in practice, non-linearity of the magnetic field gradients exists that must be accounted for or significant geometric errors occur in the volumetric image data [4].

It is worthwhile pointing out the advantages and disadvantages of 2-D and 3-D image segmentation since both are available in the system via an integrated multidimensional level set kernel [3]. For example, for small regions of interest with high signal to noise acquired with specialized surface coils (e.g. the carotid bifurcation) direct 3-D reconstruction techniques are compelling. In contrast, in the case studies discussed herein, a body coil was used (due to the large extent of the vasculature required to model surgical interventions) reducing the quality of the acquired data. The presence of complex flow and significantly diseased vessels can cause poor 
signal and vessels only a few pixels in diameter in critical regions of interest (e.g. diseased common iliac arteries). The variability in the level of the contrast agent and noise in the image data can make the constants found in the boundary velocity functions used to do the segmentation a function of position. In addition, the human body contains a vast network of arteries, but it may be desired to simulate only a subset of them. It can be difficult to extract an accurate geometric representation using the level set method in 3-D while preventing the front from advancing into undesired smaller branches (e.g. lumbar arteries). Finally, the 1-D simulation methods described in the next section require medial axis paths and circular cross section segments. Porcine in-vivo studies and other experiment work (see [6]) seem to indicate that that the global impact of approximating three-dimensional junctions with the lofting techniques described above are a second order effect compared to inaccuracies in boundary conditions. See section 4 for further discussion on possible improvements to the image segmentation.

A detailed discussion of the methods used to construct preoperative geometric models in this work from the MRA data can be found elsewhere [3,5]. Briefly, a multi-stage process is used where the first step involves extracting the medial axis paths for vessels of interest utilizing a semi-automatic algorithm. The 3-D data is then sampled in planes perpendicular to the vessel paths at user-selected locations. Several segmentation techniques including thresholding and level set techniques can then be performed on the planar image samples to extract the lumen boundary. A solid modeling operation referred to as lofting is then used to create a 3-D solid from the cross-sections of each vessel. A single 3-D solid representing the flow domain is then constructed by Boolean addition (union) of the individual vessel solid models.

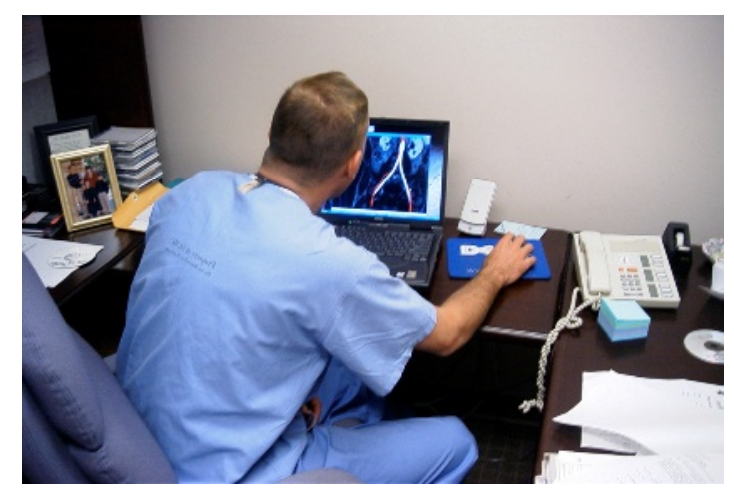

Fig. 1. The software system developed in this work was utilized by a vascular surgeon to create virtual surgical geometric models prior to the patient's actual surgery.

A surgeon can create a patient-specific surgical plan with the system using steps very similar to those used to construct the preoperative model. Specifically, the surgeon defines the path(s) the graft will follow and defines the geometry of the graft flow domain by specifying elliptical and circular cross-sections that are lofted to create a solid model. Fig. 1 shows a vascular surgeon running the software developed as part of this work on his laptop computer to create a surgical plan. 


\subsection{Boundary Condition Specification from PCMRI Data}

When MRA imaging data is acquired for the purpose of SBMP, planar slices of experimental data are also acquired providing temporally and spatially resolved velocity fields (see Fig. 3). The technique used in the present work is known as cinePhase-Contrast-Magnetic-Resonance-Imaging (PCMRI). With appropriate selection of imaging parameters, the technique can be used to quantify volumetric flow and provide insight into the spatial velocity fields in a given planar slice location. PCMRI was acquired in the case studies presented here to quantify the volumetric flow through several arteries of interest. In addition to the relatively large (with respect to pixel dimension) arteries of interest such as the aorta, aorto-femoral reconstruction planning also requires the quantification of volumetric flow in small (relative to pixel dimension) and diseased vessels such as the common iliac arteries. The software system described in [3] provides multiple segmentation and flow calculating techniques needed for SBMP.

\subsection{One-Dimensional Hemodynamic Analysis}

Post-operative volumetric flow distribution is of paramount clinical importance in alleviating the symptoms of claudication (i.e. inadequate flow to lower extremity tissue). As discussed in [6], one-dimensional finite element methods can be used to predict volumetric distributions and pressure losses. In addition to simulating resting conditions, volumetric flow distribution during exercise can be estimated by dilation and constriction of the distal vascular beds as described below.

With the assumptions that (1) blood flow velocity along the vessel axis is much greater than the flow perpendicular to the vessel axis, (2) blood can be approximated as a Newtonian fluid, and (3) the velocity profile along the axis is a scaled version of a Poiseuille cross-sectional velocity profile function, a non-linear one-dimensional equation for pulse wave propagation in elastic blood vessels has been derived [6]. The space-time finite element formulation for solving the one-dimensional problem discussed in [6] has been integrated into the present software system (see Fig. 2). The major strength of the one-dimensional method is in its speed (CPU minutes versus CPU days for three-dimensional simulation described below). However, the disadvantage of the method is that it does not account for energy losses associated with secondary flows due to curvature, branching, stenoses, aneurysms, or complex three-dimensional geometric features. As discussed in [6], minor losses due to the pressure losses across a stenosis or due to branching can be incorporated to increase the accuracy of the one-dimensional method.

An impedance boundary condition was utilized to enable simulated exercise. Briefly, a fractal like tree, based on an input root radius, length to radius ratio, and asymmetric branching factor was used to calculate an impedance function of time that produced reasonable physiologic pressures and closely matched the preoperative flow distribution determined experimentally using PCMRI [7]. By adjusting the radii of the resistance vessels in the structured tree of the viscera by constriction and dilating 
the extremities by an appropriate factor an outlet impedance approximating exercise conditions was obtained [7].

\begin{tabular}{|l|l|l|}
\hline 76 ASPIRE2 Main Menu & & \\
\hline File Path Planning Solid Modeling Meshing Boundary Conditions Simulation Display Help \\
\hline
\end{tabular}
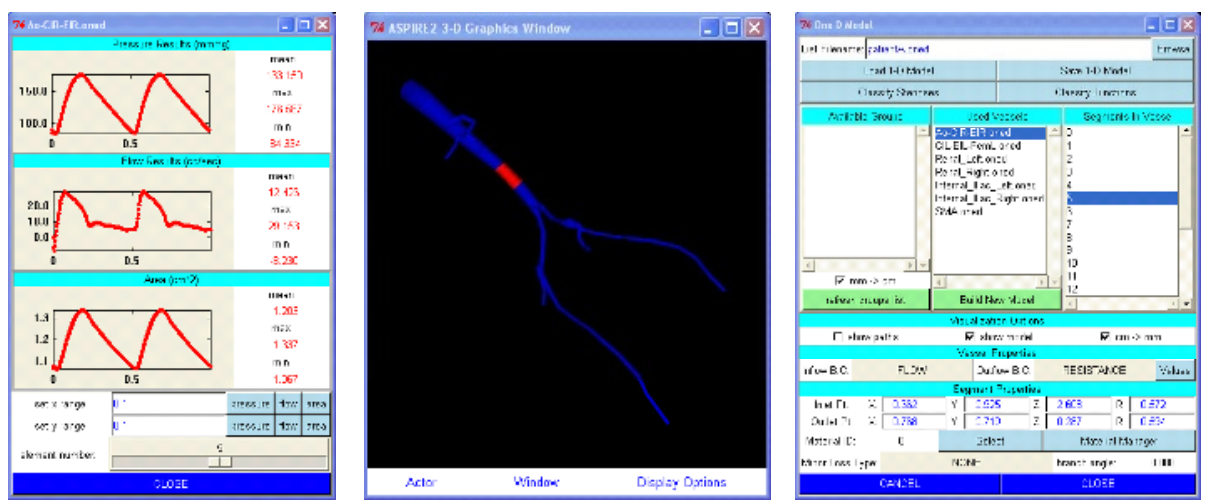

Fig. 2. An example of the graphical user interface (GUI) of the system developed for surgical planning. The "Main Menu" GUI guides a technician through the steps to go from medical imaging data to one and three dimensional hemodynamic simulation. The remaining windows in the figure are examples of controlling and running a one-dimensional analysis.

\subsection{Three-Dimensional Hemodynamic Analysis}

Mean wall shear stress and particle residence time are theorized to play an important role in disease progression. The patency of the bypass graft and long-term relief of the claudication symptoms are likely determined by flow features which can only be determined from three-dimensional analysis, while the one-dimensional analysis results provide estimates of flow distribution and pressure losses. In the threedimensional analyses presented here, due to current software limitations it was assumed that the vessel walls were rigid and blood behaves like a Newtonian fluid. The geometric models were discretized using a commercial automatic tetrahedral mesh generator (Simmetrix, Inc., Clifton Park, NY, USA). Inflow velocity boundary conditions were prescribed and volumetric outflow distributions were assigned to the arteries based on the one-dimensional analysis results. A stabilized finite element method was used to solve the Navier-Stokes equations and the results were visualized using custom software built using the Visualization Toolkit (Kitware, Inc., Clifton Park, NY, USA) [3].

\section{Results}

Two case studies demonstrate the application and limitations of the system for planning an Aorto-Femoral Reconstruction procedure. The first case involves a 67 year-old female patient while the second case involves a 55 year-old male patient. 
A preoperative and postoperative MRA for a female patient diagnosed with aortoiliac occlusive disease who underwent a direct surgical revascularization is shown in Fig. 3. Two different surgical plans were create and hemodynamic simulations were performed. Extremely low (an order of magnitude lower than the preoperative flow rates) volumetric flow was observed in the external iliac arteries during resting conditions in the simulations for the procedures shown in Fig. 3. The simulation results could explain the clinically observed occlusion of these arteries postoperatively.

(a)

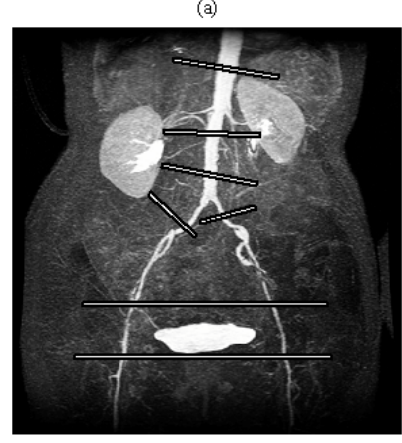

(b)

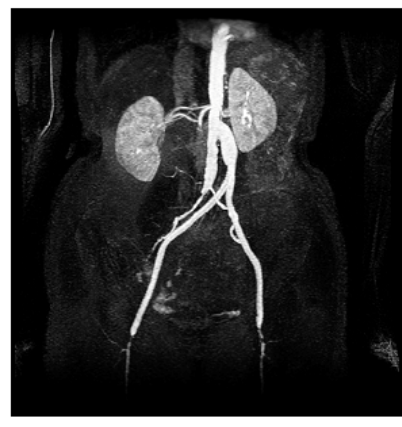

(c)

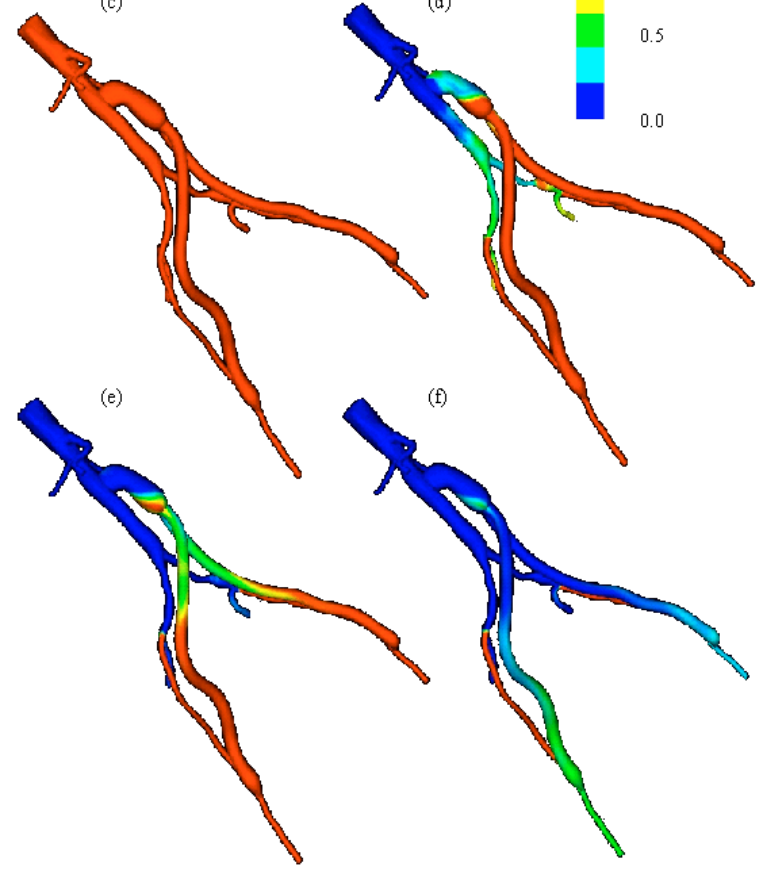

Fig. 3. 67 year-old female AFB patient. Maximum intensity projection of preoperative (a) and postoperative (b) MRA data. Seven PCMRI acquisitions were performed and the slice plane locations as indicated in (a). Notice the postoperative occlusion of the external iliac arteries in (b). A passive scalar transport problem (dye clearance time) was also solved for one of the surgical plans and this indicates area of interesting and stagnant flow (c-f).

A second case study was of a 55-year old male was experiencing severe pain in his legs during mild exercise and was diagnosed with severe aorto-iliac-femoral occlusive disease (see Fig. 4a). An end-to-side aorto-femoral bypass procedure was performed and postoperative MRA data was acquired nine days after the operation (see Fig. 4b). The postoperative MRA clearly indicated a complete occlusion of the distal native aorta. The closure of the native distal aorta was not anticipated and the simulation results for the model shown in Fig. 4 may not be consistent with the occlusion. There are two possibilities to explain the apparent inconsistency of the simulation results with the observed clinical outcome. First, the outflow boundary conditions may not 
have adequately captured the postoperative flow distribution. Second, it is possible that when the surgeon clamped the native aorta during the operation, thrombus or plaque was dislodged and traveled downstream impeding flow in the distal native aorta. This example illustrates the need for experimental validation studies before SBMP can be used clinically.

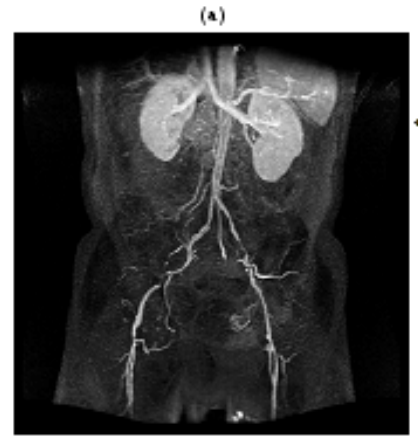

(b)

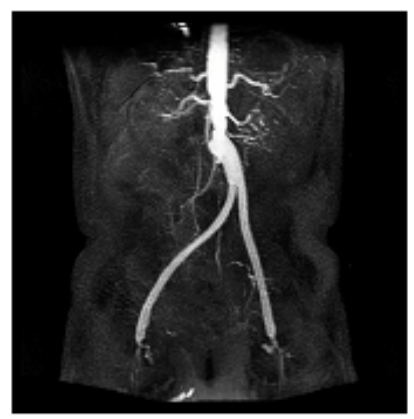

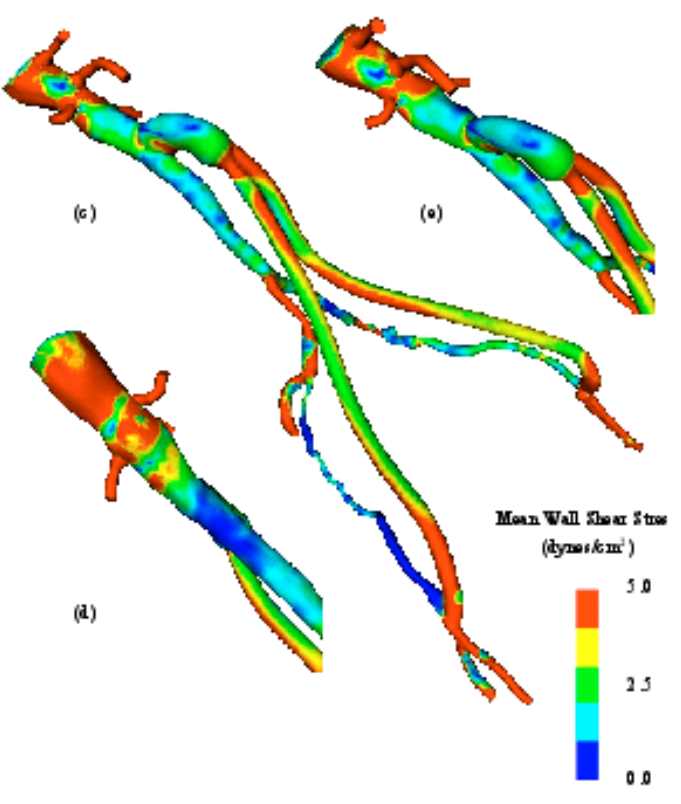

Fig. 4. 55 year-old male AFB patient. Maximum intensity projection of preoperative (a) and postoperative (b) MRA. Evaluating the long-term efficacy of a surgical procedure may require special post-processed quantities such as mean wall shear stress (c-e). Notice that postoperatively the native distal aorta occluded completely.

\section{Conclusions and Future Work}

The results presented here demonstrate the first examples of performing the geometric modeling necessary for SBMP in a clinically relevant time frame. Specifically, two case studies were shown where a vascular surgeon was able to preoperatively create geometric models representing alternative surgical procedures for a patient in approximately one hour per plan. In addition, the system enabled the processing of PCMRI data for prescribing velocity boundary conditions needed for hemodynamic simulation. Finally, in certain cases the simulation results appear to be consistent with clinically significant observed postoperative outcomes. 
The results of this work indicate several important potential research directions. For SBMP to be clinically applicable, both the geometric modeling and the simulation must be performed prior to the surgery. Integration of one-dimensional and threedimensional hemodynamic analysis into a common framework was critical given their corresponding tradeoffs for accuracy and computational expense. This motivates improvements in one-dimensional modeling including additional complex downstream boundary conditions and improved minor loss terms for special conditions such as flow in a stenosis or aneurysm. In three-dimensions, relaxing the simplifying assumptions of rigid vessel walls and integration of impedance boundary conditions is underway. Improvements in the area of geometric modeling are focused on integrating 3-D image segmentation and utilizing additional imaging modalities (i.e. CTA) in the SBMP system. Finally, additional experimental validation studies are essential to improve the numerical models and quantify the accuracy of these predictive methods.

\section{References}

1. Rutherford, R.B. (ed.): Vascular Surgical Procedures. W.B. Saunders Company, Philadelphia, PA (2000)

2. Taylor, C.A., Draney, M.T., Ku, J.P., Parker, D., Steele, B.N., Wang, K., Zarins, C.K.: Predictive Medicine: Computational Techniques in Therapeutic Decision-Making. Computer Aided Surgery, Vol. 4. (1999) 231-247

3. Wilson, N.M.: Geometric Algorithms and Software Architecture for Computational Prototyping: Applications in Vascular Surgery and MEMS. PhD Dissertation, Department of Mechanical Engineering, Stanford University, Stanford, CA USA (2002)

4. Draney, M.T., Alley, M.T., Tang, B.T., Wilson, N.M., Herfkens, R.J., Taylor, C.A.: Importance of 3D Nonlinear Gradient Corrections for Quantitative Analysis of 3D MR Angiographic Data. ISMRM (2002)

5. Wilson, N.M., Wang, K., Dutton, R.W., Taylor, C.A.: A Software Framework for Creating Patient Specific Geometric Models from Medical Imaging Data for Simulation Based Medical Planning of Vascular Surgery. MICCAI (2001) 449-456

6. Wan, J, Steele B., Spicer, S.A., Strohband, S., Feijoo, G.R., Hughes, T.J.R., Taylor, C.A.: A One-Dimensional Finite Element Method for Simulation-Based Medical Planning for Cardiovascular Disease, Computer Methods in Biomechanics and Biomedical Engineering, Volume 5, Number 3, pages 195-206 (2002)

7. Steele, B.N., Taylor, C.A.: Simulation of Blood Flow in the Abdominal Aorta at Rest and During Exercise Using a 1-D Finite Element Method with Impedance Boundary Conditions Derived from a Fractal Tree. Summer Bioengineering Meeting, Key Biscayne, FL, June 2429 , pages 813-814 (2003) 